Cita: Aguinaga, I.; Herrero-Fernández, D.; Santamaría, T. (2021). Factor protector de las estrategias de afrontamiento y la cohesión de grupo sobre el bienestar psicológico ante situaciones de ansiedad competitiva en futbolistas. Cuadernos de Psicología del Deporte, 21(1), 86-101

\title{
Factor protector de las estrategias de afrontamiento y la cohesión de grupo sobre el bienestar psicológico ante situaciones de ansiedad competitiva en futbolistas
}

\section{Protective factor of coping strategies and group cohesion on psychological well-being when facing situations of competitive anxiety in football players}

\section{Fator protetor das estratégias de enfrentamento e coesão do grupo no bem-estar psicológico diante de situações de ansiedade competitiva em jogadores de futebol}

\author{
Aguinaga, $\mathrm{I}^{1}$., Herrero-Fernández, $\mathrm{D}^{2}$., Santamaría, $\mathrm{T}^{1}$. \\ ${ }^{1}$ Universidad de Deusto, ${ }^{2}$ Universidad Europea del Atlántico
}

\begin{abstract}
RESUMEN
El bienestar psicológico que experimenta un individuo puede verse afectado por diversas variables, como, por ejemplo, la ansiedad. En el caso concreto de los deportistas, la ansiedad derivada de la práctica deportiva es algo frecuente, pudiendo derivar en niveles bajos de bienestar. Sin embargo, podrían existir factores protectores que amortiguasen esta relación. En este sentido, el objetivo del presente estudio es analizar el posible efecto protector tanto de las estrategias de afrontamiento (evaluadas mediante el Cuestionario de Estrategias de Afrontamiento en Competición Deportiva), como de la cohesión de grupo (evaluada mediante el Cuestionario de Entorno de Grupo) sobre el bienestar psicológico (evaluado mediante la Escala de Bienestar Psicológico de Ryff), a pesar de experimentar ansiedad en la competición deportiva (evaluada mediante el Cuestionario de Causas, Manifestaciones y Estrategias de Afrontamiento de la Ansiedad en la Competición Deportiva). Para ello se contó con una muestra de 99 futbolistas amateurs. Los resultados mostraron relaciones bivariadas negativas entre ansiedad y bienestar $(\mathrm{r}=-.03$ / -.37). Sin embargo, al analizar el efecto moderador tanto de la cohesión grupal $(\beta=.82, \mathrm{p}<.001)$ como de las estrategias de afrontamiento $(\beta=.87, \mathrm{p}<.001)$, se observó que ambas variables amortiguaban el efecto negativo de la ansiedad sobre el bienestar. Estos resultados pueden tener importantes implicaciones prácticas en el desarrollo de intervenciones con deportistas para mejorar el nivel de bienestar psicológico a través de la mejora tanto de la cohesión grupal como de las estrategias de afrontamiento
\end{abstract}

Palabras clave: bienestar psicológico; ansiedad competitiva; estrategias de afrontamiento; cohesión de grupo.

\section{ABSTRACT \\ The psychological well-being experienced by an individual can be affected by several variables, such as anxiety. In the specific case of athletes, anxiety derived from practicing sports is common and can lead to low levels of well- being. However, there could be protective factors that would buffer this relationship. The aim of the present study is to analyse the possible protective effect of both coping strategies (assessed with the Cuestionario de Estrategias de}




\section{Afrontamiento y cohesión en futbolistas}

Afrontamiento en Competición Deportiva) and group cohesion (assessed with the Cuestionario de Entorno de Grupo) on psychological well-being (assessed with the la Escala de Bienestar Psicológico de Ryff), despite experiencing anxiety in sports competition (assessed with the Cuestionario de Causas, Manifestaciones y Estrategias de Afrontamiento de la Ansiedad en la Competición Deportiva). A sample of 99 amateur football players was used. The results showed negative bivariate relationships between anxiety and well-being $(r=-.03 /-.37)$. However, when analysing the moderating effect of both group cohesion $(\beta=.82, \mathrm{p}<.001)$ and coping strategies $(\beta=.87, \mathrm{p}<.001)$, it was observed that both variables dampened the negative effect of anxiety on well-being. These results may have important practical implications in the development of interventions with athletes to improve the level of psychological well-being through the improvement of both coping strategies and group cohesion.

Keywords: psychological well-being, competitive anxiety, coping strategies, group cohesion.

\section{RESUMO}

O bem-estar psicológico que um indivíduo experimenta pode ser afetado por diversas variáveis, como a ansiedade. No caso específico dos atletas, a ansiedade derivada do esporte $\mathrm{O}$ bem-estar psicológico que um indivíduo experimenta pode ser afetado por diversas variáveis, como a ansiedade. No caso específico dos atletas, a ansiedade derivada do esporte é comum e pode levar a baixos níveis de bem-estar. No entanto, pode haver fatores de proteção que amenizem tal associação. Nesse sentido, o objetivo deste estudo é analisar o possível efeito protetor das estratégias de enfrentamento (avaliadas pelo Questionário de Estratégias de Enfrentamento em Competições Esportivas ) e da coesão grupal (avaliada pelo Questionário Entorno Grupal) no bem-estar psicológico dos atletas (avaliado pela Escala de Bem-estar Psicológico de Ryff), apesar da ansiedade nas competições esportivas (avaliada pelo Questionário das Causas, Manifestações e Estratégias de Enfrentamento da Ansiedade na Competição Esportiva). Para isso, investigou-se uma amostra de 99 jogadores de futebol amador. Os resultados revelaram relações bivariadas negativas entre ansiedade e bem-estar $(\mathrm{r}=-.03 /-.37)$. No entanto, ao analisar o efeito moderador das estratégias de coesão $(\beta=.82, \mathrm{p}<.001)$ e de enfrentamento em grupo $(\beta=.87, \mathrm{p}<.001)$, observou-se que ambas as variáveis atenuaram o efeito negativo da ansiedade no bem-estar. Esses resultados podem ter implicações práticas importantes no desenvolvimento de intervenções com atletas para melhorar o nível de bem-estar psicológico através do aprimoramento das estratégias de coesão e de enfrentamento em grupo.

Palavras chave: bem-estar psicológico, ansiedade competitiva, estratégias de enfrentamento, coesão de grupo

\section{INTRODUCCIÓN}

A partir de los trabajos de Seligman (Seligman y Csikszentmihalyi, 2000) se va a desarrollar un nuevo campo dentro de la psicología, denominado 'Psicología Positiva'. Esta nueva disciplina, va a permitir cambiar el foco de atención desde los efectos que la enfermedad o estados negativos de la salud mental ejercen sobre la salud en general del individuo, a enfoques más positivos que se centren en los efectos que conceptos relacionados con el bienestar o la felicidad ejercen en la salud. Este efecto, también se produce en el campo de la psicología del deporte (López-Walle, 2020), de modo que el énfasis tradicional en las emociones negativas, se traslada al campo de las emociones positivas en un área de investigación prometedor, debido, en gran parte, a la influencia que pueden tener en la mejora del rendimiento de los deportistas (McCarthy, 2011).
De esta forma, y como señalan en su estudio CantónChirivella, Checa-Esquiva y Vellisca-González (2015), es común encontrar investigaciones que aludan a conceptos positivos como las estrategias de afrontamiento adaptativas (Chirivelia y Esquiva, 2012), los estados de flow (Koehn, 2013) o, el bienestar psicológico (Smith, Ntoumanis, Duda y Vansteenkiste, 2011) en la disciplina de la psicología deportiva. De hecho, este concepto de bienestar psicológico ha adquirido gran importancia en los últimos años, dado que se ha observado cómo la práctica deportiva no sólo se relaciona con un mayor funcionamiento físico (Moreno-Fernández et. al., 2019; Olmedilla et al., 2018), sino que también contribuye a un mayor nivel de bienestar subjetivo en las personas (Berger y Tobar, 2011).

A nivel histórico, en lo que se refiere a la conceptualización y estudio del bienestar y la felicidad 


\section{Aguinaga, I.; Herrero-Fernández, D.; Santamaría, T.}

cabe distinguir dos grandes tradiciones: hedónica y eudamónica (Ryan y Deci, 2001). Desde la perspectiva hedónica, se entiende que el bienestar y la felicidad dependen esencialmente de las experiencias de placer frente a displacer, incluyendo todos los juicios acerca de aquellos elementos buenos y malos de la vida (Carrasco, Garcia-Mas y Brustad, 2009). Por su parte, la perspectiva eudamónica no equipara la felicidad subjetiva con el bienestar, basándose en que no todos los deseos de una persona se traducen en bienestar al conseguirlos (Ryan y Deci, 2001). De esta forma, Waterman (1993) resalta que desde la concepción eudamónica se invita a las personas a vivir de acuerdo con su verdadero ser, es decir, la eudamonia ocurrirá en el momento en el que las actividades de una persona sean congruentes con sus valores más profundos, y se encuentren, completamente involucradas.

A nivel psicológico, estas dos tradiciones subyacen a la distinción entre el concepto de bienestar subjetivo, vinculado al estudio del afecto y la satisfacción con la vida (enfoque hedonista) y el concepto de bienestar psicológico vinculado al desarrollo de las capacidades y el crecimiento personal (enfoque eudamónico).

Dentro de esta perspectiva eudamónica, Carol Ryff (1989a) desarrolla un modelo de bienestar psicológico considerado como precursor de los actuales modelos de Psicología Positiva y un marco de referencia a nivel teórico y empírico en el estudio actual del bienestar humano. Ryff (1989a) propone un modelo multidimensional constituido por seis dimensiones: autoaceptación, relaciones positivas con otros, autonomía, dominio del medio, propósito en la vida y crecimiento personal. Estas dimensiones determinan, teórica y operacionalmente, aquello que se relaciona con una mejor salud emocional y física (Ryff y Singer, 2009).

A pesar de que se ha aumentado el interés acerca del papel que las emociones positivas y el bienestar psicológico tienen sobre la conducta en el ámbito deportivo, en el deporte existen una gran variedad de situaciones o estímulos que el deportista puede percibir como amenazantes o peligrosas (Weber, 2018), contribuyendo de esta manera a la expresión de la ansiedad (Dosil, 2004). De acuerdo con Cheng, Hardy y Markland (2009, p.271), "la ansiedad en el contexto deportivo se entiende como un estado psicológico desagradable en reacción al estrés percibido en relación a la realización de una tarea bajo presión".

Asimismo, la ansiedad tiene, por un lado, un componente cognitivo, que incluye preocupaciones sobre el rendimiento, falta de concentración (ToméLourido, Arce y Ponte, 2019) y, por tanto, incapacidad para mantener la atención, algunos autores la denominan ansiedad cognitiva (Paludo et. al., 2020). Por otro lado, la ansiedad tiene un componente somático, que tiene que ver con la activación de síntomas físicos como, por ejemplo, el incremento de la frecuencia cardíaca (Dosil, 2004). Asimismo, la ansiedad posee un componente emocional, por cuanto pone en marcha el sistema límbico y genera en el sujeto emociones de distinto tipo (Damasio, 2005). Conviene también diferenciar entre ansiedad rasgo y ansiedad estado. La ansiedad rasgo se refiere a la predisposición a responder con ansiedad ante una gran variedad de circunstancias que, objetivamente pueden no suponer una amenaza física o psicológica (Tanguy et al., 2018). Por su parte, la ansiedad estado se refiere a aquellas circunstancias específicas para las que el deportista responde con ansiedad, como, por ejemplo, el trascurso de la competición (Weinberg y Gould, 2018).

El modelo procesual del estrés (Sandín, 1995), relaciona el estrés con la salud a partir de siete etapas interdependientes: demandas psicosociales, evaluación cognitiva, respuesta de estrés, estrategias de afrontamiento, variables sociales, variables disposicionales y estatus de salud. En este sentido, el modelo plantea que la respuesta de estrés, ocurrirá cuando las demandas contextuales excedan los recursos que posee la persona. Asimismo, esa respuesta de estrés tendrá una influencia directa sobre la salud en el caso en el que la persona no disponga de estrategias de afrontamiento adecuadas, carezca de factores sociales y variables disposicionales que modulen la relación estrés-salud.

Teniendo en cuenta este modelo, así como la relativa frecuencia de la experiencia de la ansiedad en deportistas y su relación negativa con el bienestar psicológico (Hevilla-Merino y Castillo-Rodríguez, 2018), se plantea la necesidad de buscar variables que pudieran amortiguar este efecto. En este sentido, cabe comenzar señalando el papel de las estrategias de afrontamiento. De hecho, se ha observado como aquellos deportistas que utilizan estrategias de afrontamiento adecuadas se perciben con elevados 


\section{Afrontamiento y cohesión en futbolistas}

niveles de bienestar psicológico, independientemente de experimentar ansiedad durante la competición deportiva (Cantón Chirivella et al., 2015). De acuerdo con Lazarus y Folkman (1984) el afrontamiento hace referencia a los esfuerzos tanto cognitivos como conductuales constantemente cambiantes, que tienen el fin de controlar demandas específicas externas o internas, que son percibidas como excedentes a los recursos que posee el individuo. Estos autores diferencian dos grandes dimensiones funcionales del afrontamiento. El afrontamiento orientado a la tarea incluye aquellas acciones que tratan de manejar algunos aspectos de la situación interpretada como estresante. El afrontamiento orientado a la emoción, a su vez, tiene como objetivo modificar el significado de una situación estresante, y, en consecuencia, manejar el resultado negativo de las emociones. Algunos autores han propuesto una tercera dimensión funcional (Carver, Scheier y Weintraub, 1989; Endler y Parker, 1994), conocida como afrontamiento centrado en la distracción, que tiene en cuenta todos aquellos aspectos que se asocian con el distanciamiento y distracción mental.

Otra variable que puede influir en la relación entre ansiedad y bienestar psicológico es la cohesión de grupo. La cohesión de grupo se ha relacionado con un gran número de variables, como la ansiedad precompetitiva (Prapavessis y Carron, 1996), la autoestima, el estado de ánimo o la eficacia de grupo (Eys, Hardy, Carron, y Beauchamp, 2003). Carron, Brawley y Widmeyer (1998) definen la cohesión como 'un proceso dinámico que se refleja en la tendencia de un grupo a permanecer unidos en la búsqueda de objetivos instrumentales y/o por la satisfacción de las necesidades afectivas de sus miembros" (p. 213)". A partir de esta definición, pueden extraerse las siguientes conclusiones: los grupos pueden permanecer unidos por una variedad de factores, la cohesión puede cambiar a lo largo del tiempo, los grupos se constituyen para el logro de un objetivo concreto $\mathrm{y}$, por último, pueden existir relaciones sociales entre los componentes de un grupo (Dosil, 2004). Asimismo, es importante señalar la diferenciación entre lo que se conoce como cohesión de tarea y cohesión social. La cohesión de tarea hace referencia al trabajo conjunto de un grupo para conseguir una meta. La cohesión social, por su parte, tiene que ver con la afinidad interpersonal que sienten los miembros de un grupo (Giesenow, 2007).
Por todo ello, el presente trabajo de investigación se plantea como principal objetivo examinar el posible efecto amortiguador tanto de las estrategias de afrontamiento como de la cohesión de grupo en la relación entre ansiedad en la competición experimentada en futbolistas y el bienestar subjetivo.

\section{MATERIAL Y MÉTODOS}

\section{Participantes}

Con el objetivo de calcular el tamaño muestral mínimo necesario, se llevó a cabo un análisis de la potencia estadística a priori (potencia deseada $=90 \%$ ), esperándose encontrar un tamaño del efecto medio en el análisis de moderación. Este análisis se ejecutó mediante el software G*Power 3.1 (Faul, Erdfelder, Lang, \& Buchner, 2007). El resultado mostró que el tamaño mínimo requerido era de 85 participantes. De esta manera, la muestra total de este estudio se compuso de 99 futbolistas amateurs, y fue obtenida a través de un procedimiento de muestreo incidental, es decir, por conveniencia. Del total de la muestra 53 participantes fueron hombres, y 46 participantes fueron mujeres. La media de edad se situó en 22,89 años, con una desviación típica de 4,76 años. En lo que se refiere a los datos deportivos, se encontró un mayor número de deportistas que llevaran entrenando y compitiendo entre 10 y 15 años, y que, asimismo, entrenaran una media de 3,24 días por semana, con una desviación típica de 0,73. Finalmente, las sesiones de entrenamiento de los participantes tenían una media de duración de 1,86 con una desviación típica de 0,35.

\section{Instrumentos}

Escala de Bienestar Psicológico de Ryff (1989a).

El bienestar psicológico se evaluó a través de la Escala de Bienestar Psicológico de Ryff (1989a). En esta investigación se utilizó una versión reducida, traducida y adaptada al castellano por Díaz et al. (2006), la cual está compuesta por 29 ítems con una escala Likert de 6 puntos en el que 1 significa "Totalmente en desacuerdo" y 6 "Totalmente de acuerdo". Esta escala, compuesta, a su vez, por 6 subescalas mide el grado de bienestar que sienten los participantes a través de las siguientes dimensiones: auto-aceptación, autonomía, dominio del entorno, crecimiento personal, propósito en la vida y relaciones positivas. Las evidencias de consistencia interna encontradas en la presente muestra son aceptables, con 


\section{Aguinaga, I.; Herrero-Fernández, D.; Santamaría, T.}

valores de $\alpha$ de Cronbach de ,90 para la escala global y valores de ,77 para la subescala de autoaceptación, ,74 para relaciones positivas, ,71 para autonomía, ,71 para dominio del entorno, ,80 para propósito en la vida y ,61 para crecimiento personal.

Cuestionario de Estrategias de Afrontamiento en Competición Deportiva (Gaudreau y Blondin, 2002).

Para medir las estrategias de afrontamiento empleadas por los deportistas se utilizó la adaptación para la población española realizada por Molinero, Salguero y Márquez (2010). Dicho cuestionario incluye 31 ítems, que deben contestarse con una escala tipo Likert de 5 puntos, en el que 1 significa 'Nada" y 5 significa "Mucho". Los 31 ítems del cuestionario se dividen en 8 factores, incluyendo: análisis lógico-esfuerzo, búsqueda de apoyo, relajación, control de pensamiento, resignación, salida de emociones desagradables, distracción mental, y distanciamiento. Estos 8 factores se organizan, a su vez, en tres factores de segundo orden: afrontamiento centrado en la tarea, afrontamiento centrado en la emoción y afrontamiento centrado en la distracción. En lo que se refiere a los datos de consistencia interna, se encontraron niveles altos y aceptables en esta muestra, siendo el $\alpha$ de Cronbach de ,80 para el afrontamiento centrado en la tarea, ,76 para el afrontamiento centrado en la emoción y ,83 para el afrontamiento centrado en el distanciamiento.

Cuestionario de entorno de grupo (Group Environment Questionnaire, GEQ), de Carron, Widmeyer y Brawley (1985).

Para evaluar la cohesión de grupo percibida por los deportistas se utilizó una versión de este cuestionario adaptada al castellano por Calvo (2006). Esta escala consta de 18 ítems que evalúan cuatro dimensiones de la cohesión: atracción individual hacia el grupo-tarea, atracción individual hacia el grupo-social, integración de grupo-tarea e integración de grupo-social. Los participantes responden en una escala Likert de 5 puntos en el que 1 significa "Totalmente en desacuerdo" y 5 " Totalmente de acuerdo". Por lo tanto, puntuaciones más altas significa mayor percepción de cohesión. En lo que se refiere a la consistencia interna, se encontraron niveles aceptables en esta muestra, siendo el $\alpha$ de Cronbach de ,67 para integración de grupo tarea, ,83 para integración de grupo social, ,72 para atracción individual hacia el grupo tarea, y ,69 para atracción individual hacia el grupo social.

Cuestionario de Causas, Manifestaciones y Estrategias de Afrontamiento de la Ansiedad en la Competición Deportiva (Márquez, 1992).

Con objeto de medir la ansiedad competitiva se ha utilizado la subescala de manifestaciones de la ansiedad del Cuestionario de Causas, Manifestaciones y Estrategias de Afrontamiento de la Ansiedad en la Competición Deportiva elaborado por Márquez (1992). Esta subescala de manifestaciones de la ansiedad consta de 24 ítems que permiten evaluar cuatro dimensiones: manifestaciones fisiológicas, manifestaciones emocionales, distorsiones mentales/pérdida de concentración y preocupación. El instrumento pregunta al sujeto sobre lo que piensa, siente y hace cuando está nervioso, antes y/o durante y/o después de la competición. Los participantes responden en una escala Likert de 4 puntos, en la que 0 significa "Nunca" y 4 significa "Siempre". Se demostró, en esta muestra, una consistencia interna adecuada, con valores del $\alpha$ de Cronbach de ,64 para la escala manifestaciones fisiológicas, ,75 para manifestaciones emocionales, ,88 para distorsiones mentales/pérdida de concentración y ,69 para preocupación.

\section{Procedimiento}

La muestra fue obtenida en marzo de 2019. La aplicación de los cuestionarios se realizó de modo electrónico mediante la aplicación de Google Forms. La participación fue anónima y voluntaria. Previo a su realización se les hizo llegar el consentimiento informado, el cual informaba del objetivo del estudio y la duración aproximada del cuestionario. En todo momento, a lo largo del procedimiento, se ha mantenido la confidencialidad de los datos y el anonimato de los participantes. Por último, se informó a los participantes de la posibilidad de abandonar el estudio en el momento en que así lo decidiesen.

La aplicación de los instrumentos se llevó a cabo en un único día. Asimismo, se ofreció la posibilidad a todos los participantes de poder acceder a los resultados de la investigación una vez finalizada.

\section{Análisis estadístico}




\section{Afrontamiento y cohesión en futbolistas}

Para responder al objetivo del presente estudio se planteó una investigación transversal, basada en el análisis de relación entre variables.

La estrategia de análisis consistió en dos partes. En la primera parte se analizó la asociación bivariada ( $\mathrm{r}$ de Pearson) entre todas las variables estudiadas. En la segunda parte se llevaron a cabo sendos modelos de moderación a través de modelado de ecuaciones estructurales (MSEM), a fin de testar las hipótesis de que las estrategias de afrontamiento (modelo 1) y la cohesión de grupo (modelo 2) amortiguan la relación entre ansiedad y bienestar en los futbolistas. Para la estimación de parámetros de este análisis SEM se empleó el método de máxima verosimilitud robusta, dado que el test de Mardia mostró el incumplimiento de la normalidad multivariada en ambos modelos, al ser el coeficiente superior a 6 (Mardia $=38.00$ en modelo 1; Mardia $=25.01$ en modelo 2). Este análisis se llevó a cabo mediante el software EQS 6.1 (Bentler, 2005).

Siguiendo los estándares del análisis de moderación, primeramente, se procedió a estandarizar las variables independientes y moderadoras $(\mathrm{M}=0, \mathrm{DT}=1)$, para facilitar la interpretación de los términos de interacción (Frazier, Tix, y Barron, 2004). Debido a que en ambos casos existían varias variables independientes, varias moderadoras y varias dependientes, se siguió el método de Ping para ejecutar análisis de moderación basado en la técnica SEM (Ping, 1995). Este método implica la utilización, además de la variable independiente y la moderadora correlacionadas, de una tercera variable latente no correlacionada con las anteriores que contiene un indicador a modo de producto entre los indicadores de las variables independientes y los indicadores de las variables moderadoras. Este indicador, que representa el término de interacción propio de la moderación, se crea multiplicando la suma de las puntuaciones de los indicadores de la variable dependiente por la suma de los indicadores de la variable moderadora. Para una explicación más detallada sobre los fundamentos teóricos y matemáticos sobre este método y otros diferentes para llevar a cabo análisis de moderación mediante SEM se recomienda la lectura de Cortina, Chen, y Dunlap (2001).

Los resultados del modelo completo de moderación se valoraron a través del ajuste global del modelo. Para ello, se calcularon los siguientes índices: índice de ajuste de parsimonia, el cociente entre $\square 2$ y los grados de libertad del modelo, cuyo valor ha de ser inferior a 2 para poder considerarse un ajuste adecuado (Brooke, Russell, y Price, 1988). Como índice absoluto, el Root Mean Square Error of Approximation (RMSEA) y su intervalo de confianza al $90 \%$, cuyo valor ha de ser inferior a .08 (Browne y Cudeck, 1993). Como índices incrementales, el Comparative Fit Index (CFI) y el Non-Normed Fit Index (NNFI), cuyos valores han de ser superiores a .90 (Bentler y Bonnet, 1980). Por otro lado, se analizó la significación de los coeficientes beta, en especial del término de interacción con la variable latente dependiente, lo cual determina la existencia o no de efecto de moderación.

\section{RESULTADOS}

En primer lugar, se calculó la matriz de correlaciones entre todas las variables del estudio. Los resultados se detallan en la Tabla 1. Tal como se puede observar, en líneas generales tanto las variables de ansiedad como las de afrontamiento y las de cohesión de grupo correlacionaron con las de bienestar significativamente. Por otro lado, las variables de ansiedad fueron mayoritariamente independientes de las de cohesión de grupo (relaciones no significativas), mientras que en el caso de las variables de afrontamiento mostraron correlaciones positivas y significativas con el subtipo afrontamiento hacia la emoción. Finalmente, las variables de cohesión y de afrontamiento fueron mayoritariamente independientes (relaciones no significativas).

En base a los resultados previos obtenidos, se plantearon los dos modelos de moderación. Inicialmente se analizaron las posibles diferencias por sexo en las variables de bienestar, a fin de considerar esta variable como covariante en los modelos en caso de obtener resultados estadísticamente significativos. Sin embargo, no se observaron diferencias significativas en ninguna de las seis variables: Autoaceptación $(\mathrm{t}=1.59, \mathrm{p}=.115)$, Relaciones positivas $(\mathrm{t}=-0.55, \mathrm{p}=.581)$, Autonomía $(\mathrm{t}=-0.003$, $\mathrm{p}=.998)$, Dominio del entorno $(\mathrm{t}=0.46, \mathrm{p}=.648)$, 
Aguinaga, I.; Herrero-Fernández, D.; Santamaría, T.

Tabla 1. Correlaciones bivariadas ( $\mathrm{r}$ de Pearson) entre las variables estudiadas

\begin{tabular}{|c|c|c|c|c|c|c|c|c|c|c|c|c|c|c|c|c|}
\hline & 1 & 2 & 3 & 4 & 5 & 6 & 7 & 8 & 9 & 10 & 11 & 12 & 13 & 14 & 15 & 16 \\
\hline 1. An. Fisiológica & - & & & & & & & & & & & & & & & \\
\hline 2. An. Emocional & $.61 * * *$ & - & & & & & & & & & & & & & & \\
\hline 3. An.Distor. Ment. & $.64 * * *$ & $.70 * * *$ & - & & & & & & & & & & & & & \\
\hline 4. $†$ An. Preocupac. & $.68 * * *$ & $.53 * * *$ & $.59 * * *$ & - & & & & & & & & & & & & \\
\hline 5. Afron. Tarea & .09 & -.07 & .08 & $.22 *$ & - & & & & & & & & & & & \\
\hline 6. $†$ Afron. Emoción & $.41 * * *$ & $.44 * * *$ & $.26 * *$ & $.30 * *$ & -.07 & - & & & & & & & & & & \\
\hline 7. $\dagger$ Afron. Distracc. & .10 & .15 & $.24 *$ & .15 & $.36 * * *$ & $.25^{*}$ & - & & & & & & & & & \\
\hline 8. $†$ AIGS & .11 & .03 & .04 & .00 & .02 & $.20 *$ & .11 & - & & & & & & & & \\
\hline 9. AIT & .01 & -.17 & -.06 & .14 & .19 & $-.22 *$ & -.14 & $.26 * *$ & - & & & & & & & \\
\hline 10. $\dagger$ IGS & -.01 & -.06 & .07 & .01 & .13 & -.02 & -.08 & $.51 * * *$ & $.49 * * *$ & - & & & & & & \\
\hline 11. †IGT & -.07 & $-.20 *$ & -.09 & .12 & $.26^{* *}$ & -.05 & -.06 & .17 & $.63 * * *$ & $.55 * * *$ & - & & & & & \\
\hline 12.Autoaceptación & $-.24 * *$ & $-.29 * *$ & $-.37 * * *$ & -.08 & .19 & $-.27 * *$ & -.17 & .05 & $.27 * *$ & .13 & $.29 * *$ & - & & & & \\
\hline 13. Relac. Posit. & -.16 & $-.37 * * *$ & $-.25 *$ & -.08 & .07 & -.18 & $-.20 *$ & .16 & $.29 * *$ & $.28 * *$ & $.33 * * *$ & $.38 * * *$ & - & & & \\
\hline 14. †Autonomía & -.15 & $-.31 * *$ & $-.37 * * *$ & $-.26 * *$ & $-.20 *$ & -.08 & $-.21 *$ & .07 & .05 & -.02 & .15 & $.45^{* * *}$ & $.29 * *$ & - & & \\
\hline 15. $\dagger$ Dominio Ent. & $-.25 *$ & $-.30 * *$ & $-.34 * * *$ & -.10 & .10 & $-.22 *$ & $-.27 * *$ & -.06 & $.31 * *$ & .18 & $.33 * * *$ & $.69 * * *$ & $.49 * * *$ & $.44 * * *$ & - & \\
\hline 16. $\dagger$ Crecim. Pers. & .04 & -.08 & .03 & .12 & .09 & -.02 & -.03 & .07 & .18 & .14 & $.26^{*}$ & $.38 * * *$ & $.39 * * *$ & $.40 * * *$ & $.47 * * *$ & - \\
\hline 17. Propósito Vida & $-.30 * *$ & $-.33 * * *$ & $-.36 * * *$ & -.10 & $.21^{*}$ & $-.28 * *$ & -.17 & -.12 & $.29 * *$ & .15 & $.23^{*}$ & $.75^{* * *}$ & $.32 * *$ & $.28 * *$ & $.71 * * *$ & $.34 * * *$ \\
\hline
\end{tabular}

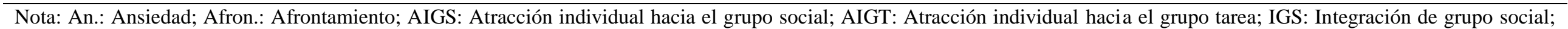
IGT: Integración de grupo tarea; Relac. Posit.: Relaciones Positivas; Dominio Ent.: Dominio Entorno; Crecim. Pers.: Crecimiento Personal.

Las variables precedidas del símbolo “†” se distribuyeron normalmente, según la prueba de D’Agostino (1986).

$* p<.05, * * p<.01, * * * p<.001$ 


\section{Afrontamiento y cohesión en futbolistas}

Crecimiento personal $(\mathrm{t}=-1.17, \mathrm{p}=.246)$, y Propósito en la vida $(t=1.57, p=.119)$. De esta manera, se analizó el efecto moderador de las estrategias de afrontamiento, de manera que el modelo resultante alcanzó un ajuste global satisfactorio según todos los índices valorados: $\chi 2 / \mathrm{gl}=1.30$, RMSEA $=.055$ (I.C. 90\%: $.004-.085)$, CFI $=.95$, NNFI $=.93$. Los resultados del modelo global se pueden observar en la Figura 1. Tal como se puede observar, aunque las estrategias de afrontamiento no estuvieron significativamente asociadas con el bienestar, el término de interacción creado a partir de las variables de ansiedad y de las variables de estrategias de afrontamiento estuvo relacionado significativamente con el factor latente correspondiente al bienestar. Esto demuestra la existencia de un efecto significativo de moderación. De esta forma, para analizar los efectos condicionales de esta moderación se representaron gráficamente los efectos. Los resultados se detallan en la Figura 2. Tal como se puede observar, los casos que presentaban un nivel alto de afrontamiento, presentaban niveles muy similares de bienestar independientemente del nivel de ansiedad. Por el contrario, los deportistas con un nivel bajo de estrategias de afrontamiento presentaron niveles sensiblemente más bajos de bienestar cuando también se daban niveles altos de ansiedad que cuando éstos eran bajos.

Seguidamente se llevó a cabo el segundo modelo de moderación. Analizando el efecto de la cohesión de grupo. En este caso el modelo mostró igualmente un ajuste satisfactorio a los datos: $\chi 2 / \mathrm{gl}=1.26$, RMSEA $=.052$ (I.C. $90 \%: .000-.080), \mathrm{CFI}=.95, \mathrm{NNFI}=.94$. Los resultados del modelo se detallan en la Figura 3. Tal como se puede observar, tanto el factor latente correspondiente a la variable independiente (ansiedad) como el factor latente correspondiente a la variable moderadora (cohesión grupal) estuvieron significativamente relacionadas con el factor latente correspondiente a la variable dependiente (bienestar). Además, el término de interacción obtuvo una relación significativa con este último, mostrando un efecto significativo de moderación. Al igual que en el anterior caso, para analizar los efectos condicionales de esta moderación se representaron gráficamente los efectos. Los resultados se detallan en la Figura 4. Tal como se puede observar, los casos que presentaban un nivel alto de cohesión grupal, presentaban niveles muy similares de bienestar independientemente del nivel de ansiedad. Por el contrario, los deportistas con un nivel bajo de cohesión grupal presentaron niveles sensiblemente más bajos de bienestar cuando también se daban niveles altos de ansiedad que cuando éstos eran bajos.

\section{DISCUSIÓN}

En la línea de las investigaciones previas centradas en los efectos que conceptos relacionados con el bienestar o la felicidad tienen en la salud (Seligman y Csikszentmihalyi, 2000), el objetivo de la presente investigación consistió en examinar el posible efecto amortiguador tanto de las estrategias de afrontamiento como de la cohesión de grupo sobre el bienestar psicológico, a pesar de experimentar ansiedad en la competición deportiva.

ansiedad (en negativo), estrategias de afrontamiento (en positivo) y cohesión de grupo (en positivo) correlacionaron, en general, de manera significativa con las variables de bienestar psicológico. A partir de estos resultados, se plantearon dos modelos de moderación que permitieron observar el factor protector de las estrategias de afrontamiento y de la cohesión de grupo sobre el bienestar psicológico en situaciones de ansiedad competitiva. Los resultados mostraron un efecto de moderación en el que en presencia de las variables de estrategias de afrontamiento y de cohesión de grupo, se debilita el efecto negativo que tiene la ansiedad sobre el bienestar psicológico.

De hecho, la relación entre las estrategias de afrontamiento y el bienestar psicológico (Urzola, Vidal, Benitez y Sañudo, 2018), así como el papel protector de las estrategias de afrontamiento en la relación entre ansiedad competitiva y bienestar psicológico, ya ha sido investigado por otros autores (Cantón Chirivella et al., 2015) obteniendo resultados similares a los señalados en la presente investigación. De igual forma, investigaciones previas ya han mostrado como percepciones elevadas de cohesión de grupo se relacionan con menores niveles de ansiedad en deportistas (Oh y Gill, 2017). En el presente estudio, se contribuye a la literatura presentando el efecto moderador de las estrategias de afrontamiento y la cohesión de grupo, de manera que ambas variables amortiguan el efecto negativo de la ansiedad sobre el bienestar psicológico de los deportistas. 


\section{Aguinaga, I.; Herrero-Fernández, D.; Santamaría, T.}

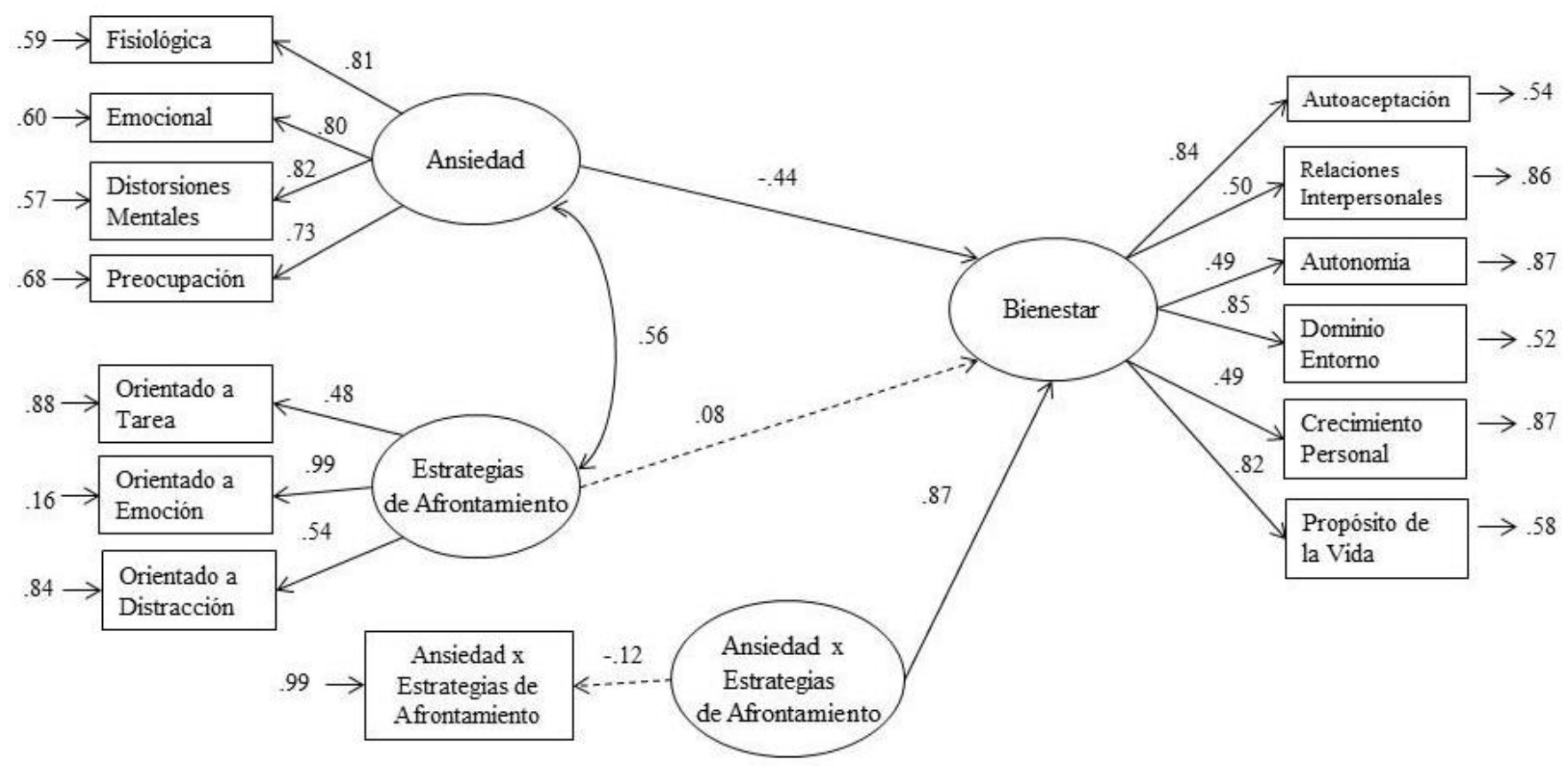

Figura 1. Modelo de moderación de las estrategias de afrontamiento en la relación entre ansiedad y bienestar.Las líneas discontinuas indican que el coeficiente no es significativo

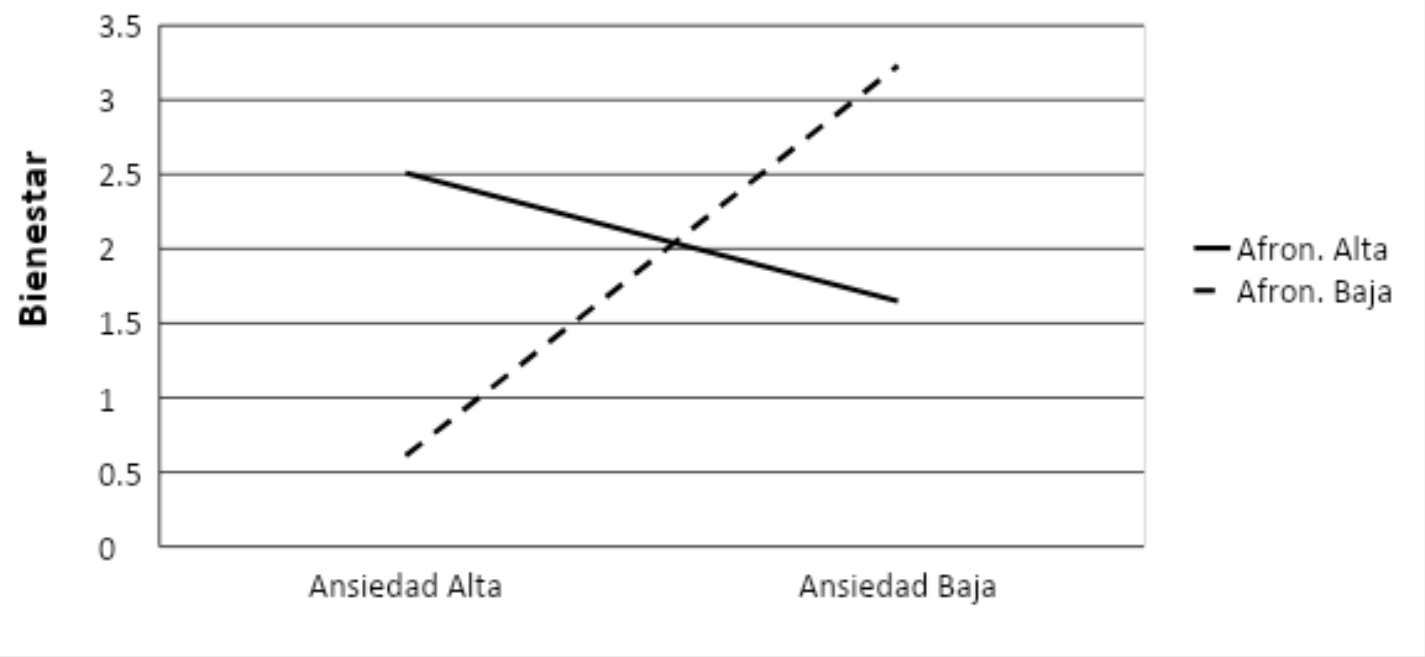

Figura 2. Análisis de los efectos condicionales de la moderación de las estrategias de afrontamiento en la relación entre la ansiedad y el bienestar 


\section{Afrontamiento y cohesión en futbolistas}

En lo que se refiere a los resultados obtenidos, se ha observado, en primer lugar, que tanto las variables de

A partir de estos resultados, y considerando el modelo procesual del estrés (Sandín, 1995), esta investigación contribuye a la comprensión de la relación entre la ansiedad y la salud de las personas. De hecho, de acuerdo con la presente investigación, en el contexto deportivo no sólo debe considerarse la relación directa existente entre ansiedad y salud, ya que esta relación se encuentra moderada por las estrategias de afrontamiento y la cohesión de grupo. Tanto las estrategias de afrontamiento como la cohesión de grupo amortiguan el efecto negativo de la ansiedad sobre el bienestar, y contribuye a que los deportistas tengan niveles altos de bienestar con independencia de los niveles de ansiedad percibidos.

Por todo ello, estos resultados deben orientar la promoción de programas de desarrollo de competencias dirigidos a deportistas, promoviendo el desarrollo de estrategias personales de afrontamiento, que posibiliten minimizar el efecto negativo de la ansiedad. También en estos programas se ve la necesidad de incorporar una mirada sistémica, incorporando las competencias relacionales que van a ofrecer al deportista recursos personales para mantener unas adecuadas relaciones y, así, favorecer la cohesión de grupo. Por último, en los itinerarios formativos de entrenadores de cualquier práctica grupal deportiva, se incidirá en los elementos puestos de manifiesto en este estudio, ofreciendo a éstos las herramientas adecuadas para favorecer el bienestar psicológico de los deportistas. Todo ello pone de manifiesto la importancia de la psicología del deporte en cuanto disciplina que posibilita no sólo el bienestar de los deportistas sino una mejora en su rendimiento.

Por último, es necesario tener en cuenta ciertas limitaciones de la presente investigación, a fin de que se tengan en cuenta en futuros estudios. En primer lugar, el tamaño de la muestra es relativamente pequeña, $y$, por tanto, futuras investigaciones con mayor tamaño muestral son necesarias a fin de contrastar los resultados obtenidos. No obstante, este tamaño muestral fue superior al criterio calculado en base a la potencia estadística calculada, lo cual supone un nivel aceptable de error Tipo II. Asimismo, es importante tener en cuenta que la presente muestra corresponde únicamente a futbolistas, por lo que los resultados obtenidos no son extrapolables a otros deportes. Además, al tratarse de un diseño correlacional, los resultados no pueden ser interpretados mediante relaciones de causa-efecto, sino como relaciones entre variables que deberán ser corroboradas en futuras investigaciones con diseños metodológicos experimentales más adecuados. Finalmente, las estrategias de afrontamiento se consideraron en el análisis de moderación como un único constructo. La razón de esta decisión radicó en el hecho de que, a pesar de las diferencias teóricas y prácticas en cuando a los tres tipos de afrontamiento, la dirección de las correlaciones con las variables dependientes (bienestar) fue la misma. Además, el análisis de los efectos condicionales con tres moderadores independientes hubiese sido extremadamente complejo tanto a nivel computacional como a nivel de interpretación de resultados. No obstante, en el método de MSEM seguido (Ping, 1995) la variable representativa del efecto de interacción se calcula en base a los pesos de todas las variables observables independientes y moderadoras, con lo cual en el producto final se pondera el efecto individual de cada moderador. En cualquier caso, esta limitación inherente al diseño del presente estudio debería ser tenida en cuenta en futuras investigaciones, analizándose el papel específico de cada estilo de afrontamiento en la relación analizada entre ansiedad y bienestar en futbolistas. 


\section{Aguinaga, I.; Herrero-Fernández, D.; Santamaría, T.}

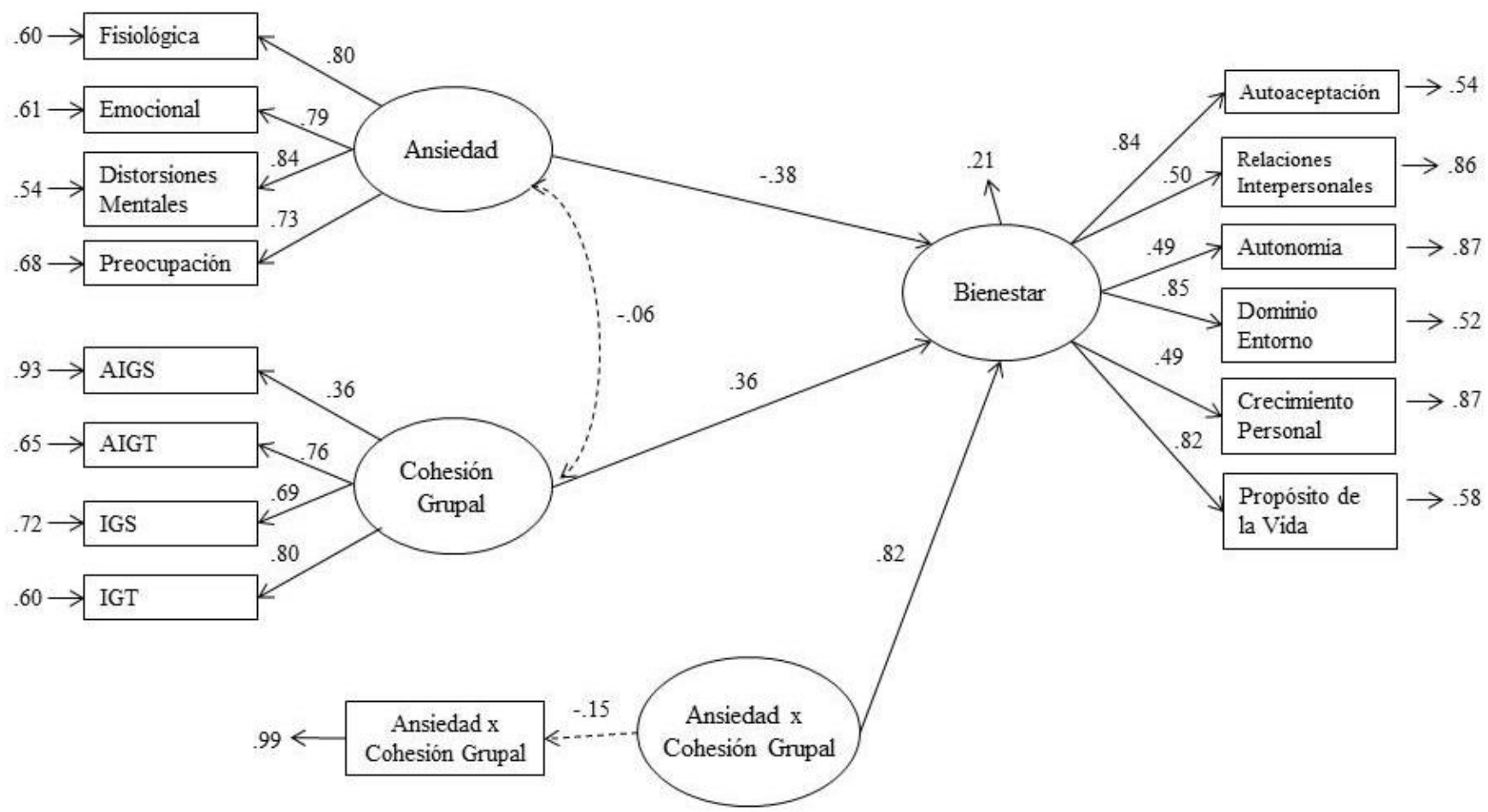

Figura 3. Modelo de moderación de las estrategias de afrontamiento en la relación entre ansiedad y bienestar. Las líneas discontinuas indican que el coeficiente no es significativo.

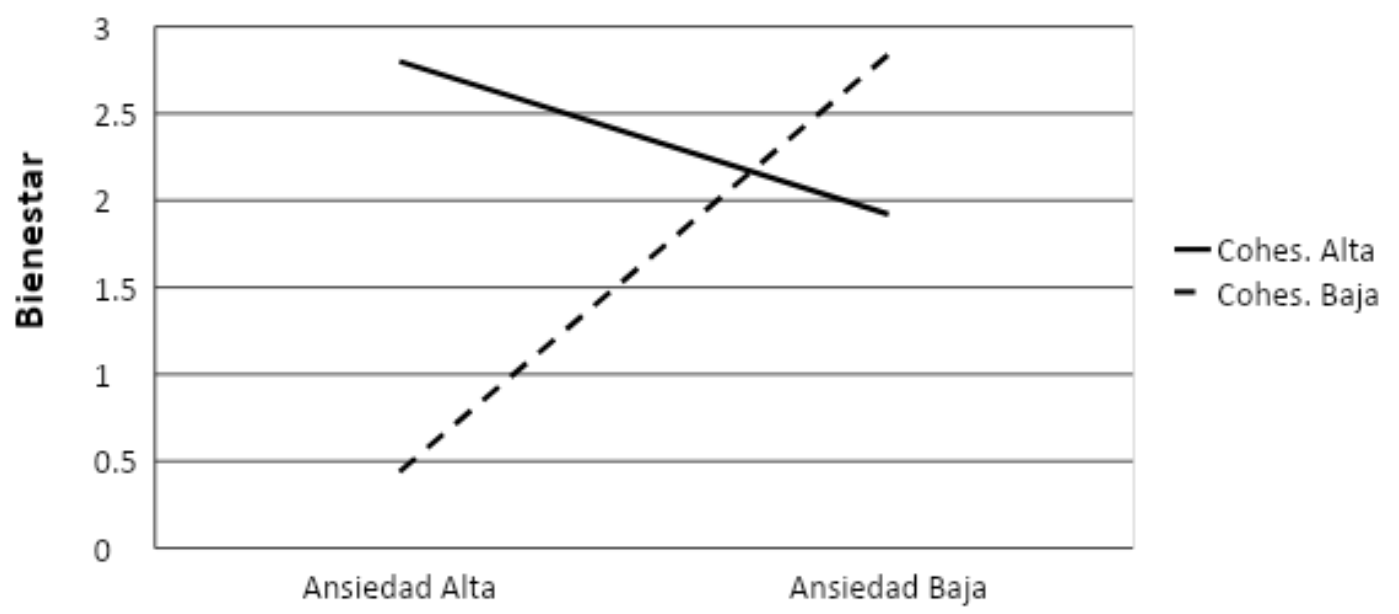

Figura 4. Análisis de los efectos condicionales de la moderación de la cohesión de grupo en la relación entre la ansiedad y el bienestar. 


\section{Afrontamiento y cohesión en futbolistas}

Por otra parte, al igual que investigaciones previas en el ámbito deportivo (Cantón Chirivella et al., 2015; Romero Carrasco et al., 2013) en el presente estudio se ha utilizado el concepto de bienestar psicológico basado en el 'Modelo Integrado de Desarrollo Personal' desarrollado por Ryff (1989b). No obstante, en estos estudios se mide el bienestar general de los participantes, y, por lo tanto, sería recomendable en futuras investigaciones desarrollar instrumentos que permitan medir el bienestar específico derivado de la práctica deportiva. Asimismo, futuros estudios podrían replicar este estudio con diferentes disciplinas deportivas, con el fin de observar si los resultados obtenidos con futbolistas, pueden ser extrapolados a otros deportes.

En cualquier caso, el presente estudio ha ofrecido información relevante sobre el papel protector tanto de las estrategias de afrontamiento como de la cohesión de grupo sobre el bienestar psicológico en deportistas que experimentan ansiedad competitiva, ayudando a la comprensión de la asociación entre estas variables. Asimismo, se han mencionado las limitaciones de la investigación, y sugerido futuras líneas de investigación que permitan conocer en mayor profundidad las relaciones entre todas estas variables.

\section{CONCLUSIONES}

La presente investigación ha puesto de relieve, en primer lugar, el impacto que tiene la ansiedad sobre el bienestar de los futbolistas. No obstante, los resultados han evidenciado el impacto amortiguador en esta relación que pueden tener las estrategias de afrontamiento y la cohesión grupal. Por tanto, trabajar de manera sistemática sobre estas dos variables en futbolistas repercutiría en el incremento del bienestar de los deportistas, independientemente del grado de ansiedad experimentado.

\section{APLICACIONES PRÁCTICAS}

Estos resultados tienen una aplicación directa en la práctica profesional. En aquellos casos en los que no puede evitarse sentir ansiedad derivada de la competición deportiva, se debe favorecer el desarrollo y la utilización en los deportistas de estrategias de afrontamiento adecuadas, así como una cohesión de grupo elevada entre los miembros del equipo deportivo. De hecho, diversas investigaciones muestran como estrategias dirigidas a aumentar las estrategias de afrontamiento y la cohesión de grupo, favorecen un estado psicológico apropiado en los deportistas, y pueden contribuir a un mayor rendimiento (Fernandez y Briceño, 2020; Wikman, Stelter, Petersen y Elbe, 2017).

\section{REFERENCIAS (APA $7^{\text {a }}$ EDICION)}

1. Bentler, P. M. (2005). EQS 6.1: Structural equations program manual. Encino, CA: Multivariate Software Inc.

2. Bentler, P. M., y Bonnet, D. G. (1980). Significance test and goodness of fit in the analysis of covariance structures. Psychological bulletin, 88, 588-606. https://doi.org/10.1037/00332909.88.3.58 8

3. Berger, B., y Tobar, D. (2011). Exercise and quality of life. In T. Morris \& P. Terry (eds.), The new sport and exercise psychology companion (pp. 438-505). Morgantown, WV: Fitness InformationTechnology.

4. Brooke, P. P., Russell, D. W., y Price, J. L. (1988). Discriminant validation of measures of job satisfaction, job involvement, and organizational commitment. Journal of Applied Psychology, 73(2), 139-145. https://doi.org/10.1037/00219010.73.2.13 9

5. Browne, M. W., y Cudeck, R. (1993). Alternative ways of assessing fit. In K. A. Bollen (Ed.), Testing Structural Equation Models (pp. 136-162). Sage: Newbury Park, CA.

6. Calvo, T. G. (2006). Motivación y comportamientos adaptativos en jóvenes futbolistas. Universidad de Extremadura.

7. Cantón-Chirivella, E., Checa-Esquiva, I., y Vellisca-González, M. Y. (2015). Bienestar psicológico y ansiedad competitiva: el papel de las estrategias de 


\section{Aguinaga, I.; Herrero-Fernández, D.; Santamaría, T.}

afrontamiento. Revista Costarricense de Psicología, 34(2), 71-78. https://doi.org/10.22544/rcps.v34i02.02

8. Carolina-Paludo, A., Nunes-Rabelo, F., Marciel-Batista, M., Rúbila-Marciel, I., Peikriszwili- Tartaruga, M., y Simöes, A.C. (2020). Game location effect on precompetition cortisol concentration and anxiety state: A case study in a futsal team. Revista de Psicología del Deporte, 29(1), 105-112.

9. Carrasco, A. E. R., Garcia-Mas, A., y Brustad, R. J. (2009). Estado del arte, y perspectiva actual del concepto de bienestar psicológico en psicología del deporte. (= State of the art and current perspective of psychological well-being in sport psychology). Revista Latinoamericana de Psicología, 41(2), 335-347.

https://doi.org/10.17060/ijodaep.2016.n2. v1.553

10. Carron, A. V., Brawley, L. R., y Widmeyer, W. N. (1998). The measurement of cohesiveness in sport groups, en J.L. Duda (ed.), Advances in sport and exercise psychology measurement, Morgantown, WV: FIT.

11. Carron, A. V., Widmeyer, W. N., y Brawley, L. R. (1985). The development of an instrument to assess cohesion in sport teams: The Group Environment Questionnaire. Journal of sport psychology, 7(3), 244-266. https://doi.org/10.1123/jsp.7.3.244

12. Carver, C. S., Scheier, M. F., y Weintraub, J. K. (1989). Assessing coping strategies: a theoretically based approach. Journal of personality and social psychology, 56(2), 267-283.

https://doi.org/10.1037/00223514.56.2.26 7

13. Cheng, W. N. K., Hardy, L., y Markland, D. (2009). Toward a three-dimensional conceptualization of performance anxiety: Rationale and initial measurement development. Psychology of Sport and Exercise, 10(2), 271-278. https://doi.org/10.1016/j.psychsport.2008 .08 .001

14. Chirivelia, E. C., y Esquiva, I. C. (2012). Los estados emocionales y su relación con las atribuciones y las expectativas de autoeficacia en el deporte TT - Emotional states and their relationship to attributions and expectations of self-efficacy in sport. Revista de Psicología Del Deporte, 21(1), 171-176. https://doi.org/10.36443/10259/204

15. Cortina, J. M., Chen, G., y Dunlap, W. P. (2001). Testing interaction effects in LISREL: Examination and illustration of available procedures. Organizational Research Methods, 4(4), 324-360. https://doi.org/10.1177/10944281014400 2

16. D'Agostino, R. B. (1986). Tests for normal distribution. In R. B. D'Agostino $\&$ M. A. Stepenes (Eds.), Goodness-of-fit techniques. New York: Macel Decker.

17. Damasio, A. (2005). En busca de Spinoza. Neurobiología de la emoción y los sentimientos. Barcelona: Crítica.

18. Díaz, D., Rodríguez-Carvajal, R., Blanco, A., Moreno-Jiménez, B., Gallardo, I., Valle, C., y Van Dierendonck, D. (2006). Adaptación española de las escalas de bienestar psicológico de Ryff. Psicothema, 18(3), 572-577.

19. Dosil, J. (2004). Psicología de la actividad física y del deporte. Madrid: McGraw-Hill.

20. Endler, N. S., y Parker, J. D. (1994). Assessment of multidimensional coping: Task, emotion, and avoidance strategies. Psychological assessment, 6(1), 50-60. https://doi.org/10.1037/1040-3590.6.1.50

21. Eys, M. A., Hardy, J., Carron, A. V., y Beauchamp, M. R. (2003). The Relationship between Task Cohesion and Competitive State Anxiety. Journal of 


\section{Afrontamiento y cohesión en futbolistas}

Sport and Exercise Psychology, 25(1), 66-76.

https://doi.org/10.1123/jsep.25.1.66

22. Faul, F., Erdfelder, E., Lang, A. G., \& Buchner, A. (2007). G*Power 3: A flexible statistical power analysis program for the social, behavioral, and biomedical sciences. Behavior Research Methods, 39(2), 175-191. https://doi.org/10.3758/bf03193146

23. Frazier, P. A., Tix, A. P., y Barron, K. E. (2004). Testing moderator and mediator effects in counseling psychology research. Journal of Counseling Psychology, 51, 115-134.

https://doi.org/10.1037/00220167.51.1.11 5

24. Gaudreau, P., y Blondin, J. P. (2002). Development of a questionnaire for the assessment of coping strategies employed by athletes in competitive sport settings. Psychology of Sport and Exercise, 3(1), 1-34. https://doi.org/10.1016/S14690292(01)00 017-6

25. Giesenow, C. (2007). Psicología de los equipos deportivos. Buenos Aires: Claridad.

26. Hevilla-Merino, A., y Castillo-Rodríguez, A. (2018). Fluctuación de las características psicológicas de rendimiento deportivo en jóvenes futbolistas. Estado basal vs Precompetitivo. Cuadernos de Psicología del Deporte, 18(3), 169-178. https://doi.org/10.4321/s1578842320130 00200003

27. Koehn, S. (2013). Effects of confidence and anxiety on flow state in competition. European Journal of Sport Science, 13(5), 543-550.

https://doi.org/10.1080/17461391.2012.7 46731

28. Lazarus, R. S. y Folkman, S. (1984). Coping and adaptation. En W. D. Gentry (Ed.), The handbook of behavioral medicine (pp. 282-325). Nueva York: Guilford.

29. López-Walle, J., Tristán, J., Tomás, I., Gallegos-Guajardo, J., Gongora, E., y Hernández-Pozo, M.R. (2020). Estrés percibido y felicidad auténtica a través del nivel de actividad física en jóvenes universitarios. Cuadernos de Psicología del Deporte, 20(2), 265-275. https://doi.org/10.6018/cpd.358601

30. Márquez, S. (1992). Adaptación española de los cuestionarios de antecedentes, manifestaciones y consecuencias de la ansiedad ante la competición deportiva. I. Estructura factorial. Revista de Psicología del Deporte, 1(2), 25-38. https://doi.org/10.4321/s1578842320140 00100007

31. McCarthy, P. J. (2011). Positive emotion in sport performance: Current status and future directions. International Review of Sport and Exercise Psychology, 4(1), 5069.

https://doi.org/10.1080/1750984X.2011.5 60955

32. Molinero, O., Salguero, A., y Márquez, S. (2010). Propiedades psicométricas y estructura dimensional de la adaptación española del Cuestionario de Estrategias de Afrontamiento en Competición Deportiva. Psicothema, 22(4), 975-983.

33. Moreno-Fernández, I.M., Gómez-Espejo, V., Olmedilla-Caballero, B., RamosPastrana, L.M., Ortega-Toro, E., y Olmedilla-Zafra, A. (2019). Eficacia de un programa de preparación psicológica en jugadores jóvenes de fútbol. Revista de Psicología Aplicada al Deporte y al Ejercicio Físico, 4(2), e14, 1-7. https://doi.org/10.5093/rpadef2019a13

34. Oh, E., \& Gill, D. (2017). An examination of the relationship between team cohesion and individual anxiety among recreational soccer players. Journal of Amateur Sport, $3(2)$, $1-26$. https://doi.org/10.17161/jas.v3i2.5883 


\section{Aguinaga, I.; Herrero-Fernández, D.; Santamaría, T.}

35. Olmedilla, A., Fuensanta SánchezAldeguer, M., Almansa, C.M., GómezEspejo, V., y Ortega, E. (2018). Entrenamiento psicológico y mejora de aspectos psicológicos relevantes para el rendimiento deportivo en jugadoras de fútbol. Revista de Psicología Aplicada al Deporte y al Ejercicio Físico, 3(1), e8, 111. https://doi.org/10.5093/rpadef2018a2

36. Ping, R. A. (1995). A parsimonious estimating technique for interaction and quadratic latent variables. Journal of Marketing Research, 32, 336-347. https://doi.org/10.1177/00222437950320 0308

37. Romero Carrasco, A. E., Brustad, R., Zapata Campbell, R., Aguayo Cuevas, C., y García Ucha, F. (2013). Bienestar psicológico y psicopatología: Estudio de un caso de intervención en el equipo técnico y deportistas de un club de tenis. Revista de psicología del deporte, 22(2), 0387-394. https://doi.org/10.5944/rppc.vol.11.num.2 .2006 .4021

38. Ryan, R. M., y Deci, E. L. (2001). On Happiness and Human Potentials: A Review of Research on Hedonic and Eudaimonic Well-Being. Annual Review of Psychology, 52(1), 141-166. https://doi.org/10.1146/annurev.psych.52. 1.141

39. Ryff, C. D. (1989a). Beyond Ponce de Leon and life satisfaction: New directions in quest of successful ageing. International journal of behavioral development, 12(1), 35-55. https://doi.org/10.1177/01650254890120 0102

40. Ryff, C. D. (1989b). Happiness is everything, or is it? Explorations on the meaning of psychological wellbeing. Journal of personality and social psychology, 57(6), 1069-1081. https://doi.org/10.1037/00223514.57.6.10 69
41. Ryff, C. D., y Singer, B. (2009). Psychological Inquiry: An International Journal for the Advancement of Psychological Theory The Contours of Positive Human Health The Contours of Positive Human Health, (June 2014), 3741.

https://doi.org/10.1207/s15327965pli090 1_1

42. Sandín, B. (1995). El estrés. En A. Belloch, B. Sandín y F. Ramos (Eds.), Manual de Psicopatología (Vol. II, pp. 352). Madrid: McGraw-Hill.

43. Seligman, M.E. y Csikszentmihalyi, M. (2000). Positive psychology An introduction. American psychologist, 55, 5-14. https://doi.org/10.1037/0003-066X.55.1.5

44. Smith, A. L., Ntoumanis, N., Duda, J. L., y Vansteenkiste, M. (2011). Goal striving, coping, and well-being: a prospective investigation of the self-concordance model in sport. Journal of Sport \& Exercise Psychology, 33(1), 124-145. https://doi.org/10.1123/jsep.33.1.124

45. Tanguy, G., Sagui, E., Fabien, Z., MartinKrumm, C., Canini, F., y Trouselard, M. (2018). Anxiety and PsychoPsychological Stress Response to Competitive Sport Exercise. Frontiers in Psychology, 27. https://doi.org/10.3389/fpsyg.2018.01469

46. Tomé-Lourido, D., Arce, C. y Ponte, D. (2019). The relationship between competitive state anxiety, self confidence and attentional control in athletes. Revista de Psicología del Deporte, 28(2), 143150.

47. Urzola, A. U., Vidal, I. R., Benítez, I. V., \& Sañudo, J. E. P. (2018). La importancia de las estrategias de afrontamiento en el bienestar psicológico en una muestra escolarizada de adolescentes. Psicogente, 21(40), 440-457. https://doi.org/10.17081/psico.21.40.308 2 


\section{Afrontamiento y cohesión en futbolistas}

48. Waterman, A. S. (1993). Two Conceptions of Happiness: Contrasts of Personal Expressiveness (Eudaimonia) and Hedonic Enjoyment. Journal of Personality and Social Psychology, 64(4), 678-691.

https://doi.org/10.1037/00223514.64.4.67 8

49. Wikman, J. M., Stelter, R., Petersen, N. K., \& Elbe, A. M. (2017). Effects of a team building intervention on social cohesion in adolescent elite football players. Swedish Journal of Sport Research.

50. Weber, S., Puta, C., Lesinski, M., Gabriel, B., Steidten, T., Bär, K., Herbsled, M., Granacher, U., y Gabriel, H. (2018). Symptoms of Anxiety and Depression in Young Athletes Using the Hospital
Anxiety and Depression Scale. Frontiers in Psychology, 182. https://doi.org/10.3389/fphys.2018.00182

51. Weinberg, R. S., y Gould, D. (2019). Foundations of Sport and Exercise Psychology, 7E. Champaign: Human Kinetics. 